\title{
Thermal redistribution of localized excitons and its effect on the luminescence band in InGaN ternary alloys
}

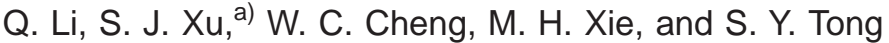 \\ Department of Physics, The University of Hong Kong, Pokfulam Road, Hong Kong, China \\ C. M. Che \\ Department of Chemistry, The University of Hong Kong, Pokfulam Road, Hong Kong, China \\ $\mathrm{H}$. Yang \\ State Key Laboratory for Integrated Optoelectronics, Institute of Semiconductors, \\ Chinese Academy of Science, Beijing, 100083, China
}

(Received 21 March 2001; accepted for publication 18 July 2001)

\begin{abstract}
Temperature-dependent photoluminescence measurements have been carried out in zinc-blende InGaN epilayers grown on GaAs substrates by metalorganic vapor-phase epitaxy. An anomalous temperature dependence of the peak position of the luminescence band was observed. Considering thermal activation and the transfer of excitons localized at different potential minima, we employed a model to explain the observed behavior. A good agreement between the theory and the experiment is achieved. At high temperatures, the model can be approximated to the band-tail-state emission model proposed by Eliseev et al. [Appl. Phys. Lett. 71, 569 (1997)]. (C) 2001 American Institute of Physics. [DOI: 10.1063/1.1403655]
\end{abstract}

One of the most characteristic features in InGaN alloys is the apparent local composition fluctuations in In and $\mathrm{Ga}$ concentrations. Generally, random alloy disorder is a common phenomenon in ternary semiconductor materials. However, in InGaN, there has been more and more evidence ${ }^{1-4}$ showing that In-rich clusters with a broad size distribution from the atomic scale up to $100 \mathrm{~nm}$ form, instead, a simple random statistical distribution of $\mathrm{In}$ and $\mathrm{Ga}$ atoms. These In-rich clusters create a complex spatial landscape for the electronic states and lead to the presence of localized states with energies of several hundred meV below the band gap. ${ }^{5}$ As a result, several anomalous phenomena, such as the coexistence of high luminescence efficiency and high density of threading dislocations as well as the blueshift of luminescence peak position with increasing temperature, etc., are quite often observed in InGaN alloys grown on different substrates with different growth techniques. A band-tail-state emission model is proposed by Eliseev et al. ${ }^{6}$ to interpret the frequently observed blueshift of the emission peak with temperature.

In this letter, temperature-dependent photoluminescence (PL) spectra of zinc-blende InGaN epilayers grown on GaAs substrate have been measured. We focus on the observed anomalous temperature dependence of the luminescence peak positions of the samples. A model, taking in account the thermal activation and transfer of the localized excitons, is developed to fit the experimental results. A good agreement between the experiment and the theory over almost the whole interested temperature range is obtained. Interestingly, the present model can be approximated to the Eliseev's model $^{6}$ at the high-temperature region.

The $\operatorname{In}_{x} \mathrm{Ga}_{1-x} \mathrm{~N}$ epilayers investigated here were grown

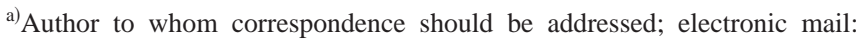
sjxu@hkucc.hku.hk
}

on $\operatorname{GaAs}(001)$ substrates with metalorganic vapor-phase epitaxy. Prior to the growth of about $0.3 \mu \mathrm{m} \mathrm{InGaN} \mathrm{layers,} \mathrm{an}$ about 0.6- $\mu \mathrm{m}$-thick zinc-blende $\mathrm{GaN}$ buffer layer was deposited. Further details of the growth can be found elsewhere. ${ }^{7}$ Two samples with In concentrations of $21 \%$ (sample A) and $14 \%$ (sample B) (Refs. 7 and 8) were investigated in the work. The PL system has been previously described elsewhere. ${ }^{9}$ The temperature of the samples ranged from 4 to $300 \mathrm{~K}$. A standard phase lock-in technique was employed to enhance the signal-to-noise ratio.

Figure 1 shows the PL spectra of sample A at several different temperatures. The peak position of the lumines-

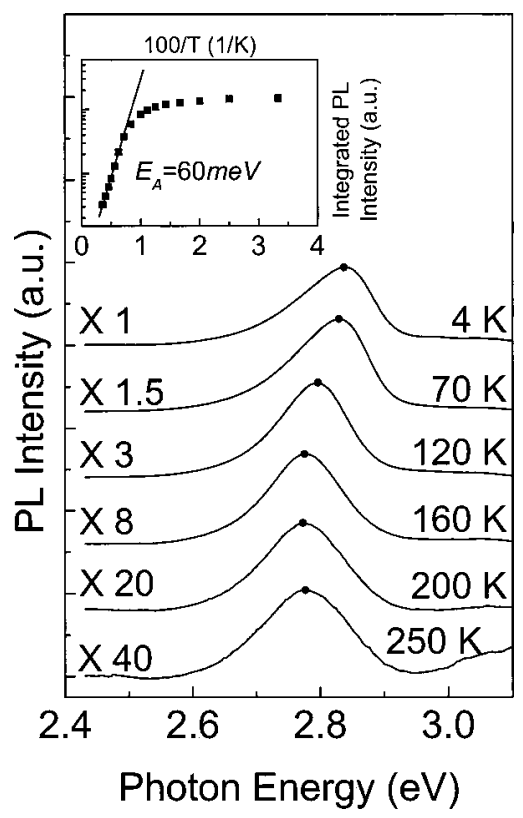

FIG. 1. PL spectra of sample $A\left(\operatorname{In}_{0.21} \mathrm{Ga}_{0.79} \mathrm{~N}\right)$ at different temperatures. The inset shows the temperature-dependent emission intensity (solid square) and the fitting curves with $I \propto e^{E_{A} / k_{B} T}$. 


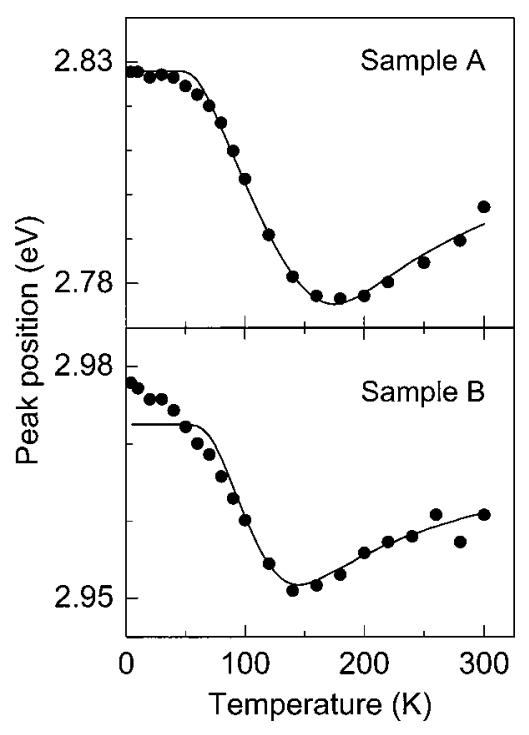

FIG. 2. Peak positions of the luminescence bands of samples A and B as a function of temperature. Solid circles are the experimental data while solid lines are the fitting results according to Eqs. (3) and (4).

cence band of sample A at low temperature is at $\sim 2.83 \mathrm{eV}$, which is in good agreement with recent theoretical results. ${ }^{10}$ The temperature dependence of the peak positions (solid circles) of the luminescence band of sample A is shown in Fig. 2. Obviously, it does not follow the behavior of a semiconductor band gap predicted by either the Varshni ${ }^{11}$ or Bose-Einstein ${ }^{12}$ formulas. When the temperature is below $160 \mathrm{~K}$, the luminescence band redshifts faster than that predicted by Varshni's empirical formula as the temperature increases. However, the blueshift of the luminescence peak takes place when the temperature is up to $160 \mathrm{~K}$. The temperature-induced blueshift of the luminescence peak has been observed in a lot of different semiconductor materials ${ }^{13,14}$ including InGaN. ${ }^{6}$ The faster redshift of the luminescence band in the low-temperature region was observed in a self-organized InAs/GaAs quantum-dot ensemble with a large size distribution. ${ }^{15}$ InGaN alloys with In-rich clusters are very similar to the InAs/GaAs quantum-dot systems. Therefore, it can be expected that there are very similar behaviors of the luminescence bands between two material systems. Starting from the rate equation proposed by $\mathrm{Xu}$ et al. ${ }^{16}$ we further derive a model to fit the observed temperature dependence of the luminescence band in zincblende InGaN alloys.

It has been demonstrated that the radiative recombination of localized excitons dominates in the luminescence processes in InGaN alloys. ${ }^{5}$ The localized electronic states is a distribution function of energy and is assumed to be a Gaussian-like function here, that is, $\rho(E) \propto \exp [-(E$ $\left.\left.-E_{0}\right)^{2} / 2 \sigma^{2}\right]$, where $E_{0}$ and $\sigma$ represent the center and width of the distribution function respectively. When the sample is heated, thermal activation and thermal transfer of the localized excitons occur. This leads to the temperature-dependent redistribution of the localized excitons. Under weak excitation conditions, the dynamics of the localized excitons can be described by the following rate equation: ${ }^{16}$ Sity of sample A is plotted in the inset of Fig. 1. The thermal
Downloaded 06 Nov 2006 to 147.8.21.97. Redistribution subject to AlP license or copyright, see http://apl.aip.org/apl/copyright.jsp

$$
\begin{aligned}
\frac{d N(E, T)}{d t}= & G(E)+\alpha \int_{E^{\prime}} \frac{N\left(E^{\prime}, T\right)}{\tau_{\mathrm{tr}}} e^{\left(E^{\prime}-E_{a}\right) / k_{B} T} d E^{\prime} \\
& \times \frac{\rho(E)}{\int_{E} \rho(E) d E}-\frac{N(E, T)}{\tau_{\mathrm{tr}}} e^{\left(E-E_{a}\right) / k_{B} T} \\
& -\frac{N(E, T)}{\tau_{r}}
\end{aligned}
$$

where $N(E, T)$ is the distribution of the localized excitons; $G(E)$ is the generation rate, which is proportional to the distribution of the localized states; $E_{a}$ represents a barrier level that the carriers must overcome to transfer; $k_{B}$ is the Boltzmann constant; $T$ is the lattice temperature; $\tau_{\text {tr }}$ is the carrier transfer time; $\tau_{r}$ is the carrier recombination time; and $\alpha$ represents the carrier recapture coefficient. Under steadystate conditions, that is, $d N(E, T) / d t=0$, the solution of Eq. (1) can be written as

$$
N(E, T) \propto \frac{e^{-\left(E-E_{0}\right)^{2} / 2 \sigma^{2}}}{e^{\left(E-E_{a}\right) / k_{B} T}+\tau_{\mathrm{tr}} / \tau_{r}} .
$$

Let $\partial N(E, T) / \partial E=0$, the peak positions of $N(E, T)$ at different temperatures can be derived from Eq. (2). We find that when

$$
E=E_{0}-x(T) k_{B} T,
$$

$N(E, T)$ reaches its maximum values. The temperaturedependent dimensionless coefficient $x(T)$ in Eq. (3) can be obtained by numerically solving the following equation:

$$
x e^{x}=\left(\frac{\tau_{r}}{\tau_{\mathrm{tr}}}\right)\left[\left(\frac{\sigma}{k_{B} T}\right)^{2}-x\right] e^{\left(E_{0}-E_{a}\right) / k_{B} T} .
$$

Equation (4) has only one solution at $0<x<\left(\sigma / k_{B} T\right)^{2}$.

If all localized excitons are assumed to have the same radiative recombination rate, the peak position of $N(E, T)$ represents the peak positions of the luminescence band. Thus, Eqs. (3) and (4) can be employed to fit the experimental data. The solid lines in Fig. 2 are the fitting results to the experimental data of samples $\mathrm{A}$ and $\mathrm{B}$, respectively. The fitting parameters are $E_{0}=2.828 \mathrm{eV}, E_{a}-E_{0}=75 \mathrm{meV}, \tau_{\text {tr }} / \tau_{r}$ $=0.022 \mathrm{ps} / 800 \mathrm{ps}$, and $\sigma=30 \mathrm{meV}$ for sample A and $E_{0}$ $=2.9725 \mathrm{eV}, \quad E_{a}-E_{0}=80 \mathrm{meV}, \quad \tau_{\text {tr }} / \tau_{r}=0.022 \mathrm{ps} / 500 \mathrm{ps}$, and $\sigma=17.2 \mathrm{meV}$ for sample B, respectively. Quite good agreement between theory and experiment is obtained. The energy difference $E_{a}-E_{0}$ reflects the magnitude of the carrier localization. We note that our values of $E_{a}-E_{0}$ are in good agreement with the localization depth in the wurtzite $\mathrm{In}_{0.15} \mathrm{Ga}_{0.85} \mathrm{~N}$ reported by Yang, Lin, and Chen ${ }^{17}$ very recently.

At the high-temperature region, when $\left(\tau_{r} / \tau_{\text {tr }}\right) \exp \left[\left(E_{0}\right.\right.$ $\left.\left.-E_{a}\right) / k_{B} T\right] \gg 1$, the approximated solution of Eq. (4) is given as

$$
x(T) \approx\left(\sigma / k_{B} T\right)^{2},
$$

and Eq. (3) becomes $E \approx E_{0}-\sigma^{2} / k_{B} T$. Then, our model becomes the band-tail-state emission model proposed by Eliseev et al. ${ }^{6}$ A comparison between Eq. (4) and Eliseev's model is given in Fig. 3, showing that the two models are consistent with each other at the high-temperature region.

The temperature dependence of the integrated PL intensity of sample A is plotted in the inset of Fig. 1. The thermal 


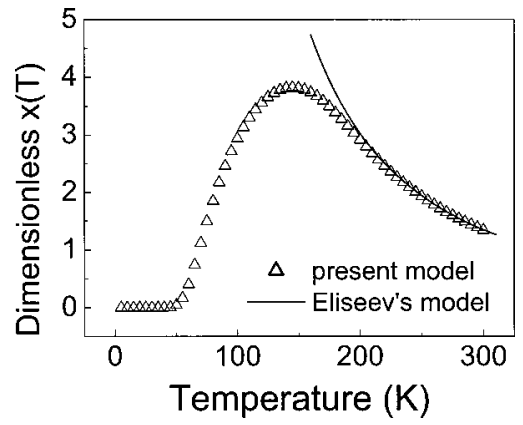

FIG. 3. Comparison between the present model and the band-tail-state emission model proposed by Eliseev et al. (see Ref. 6). using the fitting parameters of sample A.

activation energy $E_{A}$ can be obtained by fitting the data with $I \propto \exp \left(E_{A} / k_{B} T\right)$. We got $E_{A}=60 \mathrm{meV}$ for sample A and $E_{A}$ $=52 \mathrm{meV}$ for sample B, both less than the corresponding values of $\left(E_{a}-E_{0}\right)$ of the two samples. We know that at the high-temperature region, quite often impurities and defects in semiconductors are thermally activated as nonradiative centers to quench the luminescence, and consequently, reduce the thermal activation energy.

In conclusion, the PL spectra of zinc-blende InGaN alloys at different temperatures were measured. Unusual temperature dependence of PL peak energy is observed and is fitted well by a model of thermally activated redistribution within a Gaussian (energy) distribution of localized states. The results provide evidence for exciton localization up to room temperature, with the energies of the localized states described by a Gaussian distribution with a spread of 30 meV (sample A) or $17 \mathrm{meV}$ (sample B). Several researchers have suggested that In-rich clusters of varying sizes may give rise to localized exciton states with a broad distribution of energies in InGaN films.
The work was supported by HKU URC Grant No. 10203533 and partly supported by the AOE program of lightemitting materials at HKU and NSFC/RGC Joint Research Scheme No. N_HKU028/00. One of the authors (C.M.C.) acknowledges financial support from the Croucher Foundation of Hong Kong.

${ }^{1}$ K. P. O'Donnell, R. W. Martin, and P. G. Middleton, Phys. Rev. Lett. 82, 237 (1999).

${ }^{2}$ S. F. Chichibu, K. Wada, J. Mullhauser, O. Brandt, K. H. Ploog, T. Mizutani, A. Setoguchi, R. Nakai, M. Sugiyama, H. Nakanishi, K. Torii, T. Deguchi, T. Sota, and S. Nakamura, Appl. Phys. Lett. 76, 1671 (2000).

${ }^{3}$ V. Lemos, E. Silveira, J. R. Leite, A. Tabata, R. Trentin, L. M. R. Scolfaro, T. Frey, D. J. As, D. Schikora, and K. Lischka, Phys. Rev. Lett. 84, 3666 (2000).

${ }^{4}$ H. Chen, R. M. Feenstra, J. E. Northrup, T. Zywietz, and J. Neugebauer, Phys. Rev. Lett. 85, 1902 (2000).

${ }^{5}$ See, for example, a recent review article, N. M. Johnson, A. V. Nurmikko, and S. P. DenBaars, Phys. Today 53, 31 (2000).

${ }^{6}$ P. G. Eliseev, P. Perlin, J. Lee, and M. Osiski, Appl. Phys. Lett. 71, 569 (1997).

${ }^{7}$ J. B. Li, H. Yang, L. X. Zheng, D. P. Xu, and Y. T. Wang, MRS Internet J. Nitride Semicond. Res. 4S1, G3.25 (1999).

${ }^{8}$ X. L. Sun, Y. T. Wang, H. Yang, L. X. Zheng, D. P. Xu, J. B. Li, and Z. G. Wang, J. Appl. Phys. 87, 3711 (2000).

${ }^{9}$ S. J. Xu, W. Liu, and M. F. Li, Appl. Phys. Lett. 77, 3376 (2000).

${ }^{10}$ L. K. Teles, J. Furthmller, L. M. R. Scolfaro, J. R. Leite, and F. Bechstedt, Phys. Rev. B 63, 085204 (2001).

${ }^{11}$ Y. P. Varshni, Physica 34, 149 (1967).

${ }^{12}$ L. Vina, S. Logothetidis, and M. Cardona, Phys. Rev. B 30, 1979 (1984).

${ }^{13}$ L. Bergman, M. Dutta, M. A. Stroscio, S. M. Komirenko, C. J. Eiting, D. J. H. Lambert, H. K. Kwon, and R. D. Dupuis, Appl. Phys. Lett. 76, 1969 (2000), and references therein.

${ }^{14}$ S. Guha, Q. Cai, M. Chandrasekhar, H. R. Chandrasekhar, H. Kim, A. D. Alvarenga, R. Vogelgesang, A. K. Ramdas, and M. R. Melloch, Phys. Rev. B 58, 7222 (1998).

${ }^{15}$ Z. Y. Xu, Z. D. Lu, Z. L. Yuan, X. P. Yang, B. Z. Zheng, J. Z. Xu, W. K. Ge, Y. Wang, J. Wang, and L. L. Chang, Phys. Rev. B 54, 11528 (1996).

${ }^{16}$ Z. Y. Xu, Z. D. Lu, Z. L. Yuan, X. P. Yang, B. Z. Zheng, J. Z. Xu, W. K. Ge, Y. Wang, J. Wang, and L. L. Chang, Superlattices Microstruct. 23, 381 (1998).

${ }^{17}$ H. C. Yang, T. Y. Lin, and Y. F. Chen, Appl. Phys. Lett. 78, 338 (2001). 